\title{
Hedgehog inhibitor pokes tumor
}

Patients with the most common form of pancreatic cancer, ductal adenocarcinoma, barely respond to therapy. A study in mice now opens the door to a new approach. Olive et al. ${ }^{1}$ took aim at the tough layer of fibroblasts that encase such tumors and limit their accessibility to drugs. This inhibitor, in combination with a more conventional drug (gemcitabine), allowed blood vessels to penetrate such tumors and shrank them in a mouse model. The lives of the mice were extended only by a few weeks, but the approach opens the door to new ways of treating this difficult cancer.

\section{We are still limited by a lack of knowledge about the detailed molecular mechanisms driving pancreatic cancer development and maintenance.-Rune Toftgård}

\section{Michael Hollingsworth:}

The paper by Olive et al. ${ }^{1}$ highlights a common therapeutic problem that is extreme in pancreatic cancer: desmoplasia-a complex tumor microenvironment consisting of immune cells, fibroblasts, lymphatic and vascular endothelial cells-creates a tumor microenvironment that facilitates tumor growth and metastasis and blocks drug delivery through the blood vessels. Aberrant expression of the developmental morphogen Hedgehog drives such desmoplasia in pancreatic cancer ${ }^{2}$. Hedgehog is an excellent target for disrupting the protected cellular niche created by tumors for growth and survival, especially when combined with agents (such as gemcitabine) that target tumor growth.

The finding that inhibiting Hedgehog signaling in stroma increases drug delivery and decreases metastasis may also be explained in part by a recent report demonstrating that $\mathrm{SHH}$ induces lymphangiogenesis ${ }^{3}$, which affects intratumoral pressure, and may represent an important conduit for metastasis. The age is upon us of attempting rational combination therapies that target the tumor, its microenvironment and the vasculature. Taking aim at developmental pathways that regulate formation and maintenance of the full tumor microenvironment is an excellent starting point.

Professor, The Eppley Institute for Research in Cancer and Allied Diseases, University of Nebraska Medical Center, Omaha, Nebraska, USA.

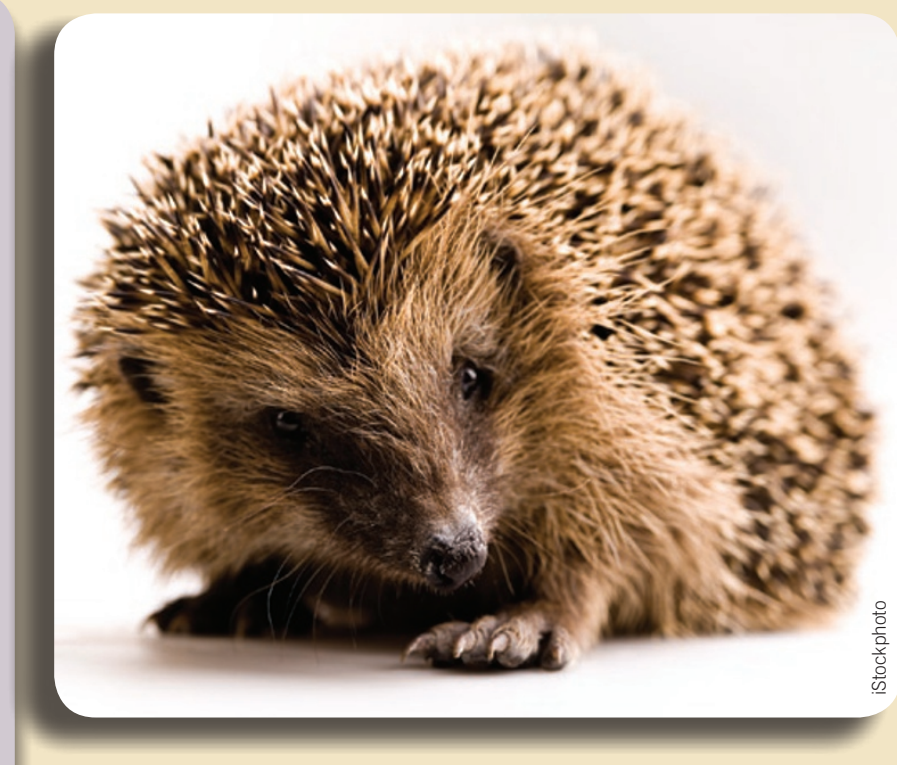

\section{Rune Toftgård:}

This study opens new avenues for the treatment of pancreatic cancer $^{1}$. We are still limited, however, by a lack of knowledge about the detailed molecular mechanisms driving pancreatic cancer development and maintenance. As one example, Hedgehog signaling in pancreatic cancer, for as yet unknown reasons, predominantly originates with the tumor, with stromal cells at the receiving end; in contrast, Hedgehog signaling in epithelial cells is required for the acinar regeneration observed in association with pancreatitis. Moreover, the Hedgehog signaling pathway is activated in a receptor-independent way in some tumor models and is enhanced in metastases. Perhaps a further benefit could be provided by inhibition of Hedgehog signaling not only in stromal cells, but also in tumor cells, through blocking of receptor-independent signaling.

Director of the Center for Biosciences, Karolinska Institutet, Stockholm, Sweden. 